\title{
FILTERING AND SEGMENTATION OF POLARIMETRIC SAR IMAGES WITH BINARY PARTITION TREES
}

\author{
Alberto Alonso-González, Carlos López-Martínez, Philippe Salembier \\ Universistat Politècnica de Catalunya (UPC), Signal Theory and Communications Dept. (TSC) \\ Jordi Girona 1-3, 08034 Barcelona, Tlf. +34 934016785 Email: alberto.alons@ gmail.com
}

\begin{abstract}
A new multi-scale PolSAR data filtering technique, based on a Binary Partition Tree (BPT) representation of the data, is proposed. Different alternatives for the construction and the exploitation of the BPT for filtering and segmentation are presented. Results with simulated and experimental PolSAR data are presented to shown the capabilities of the BPT-filtering strategy to maintain both spatial details and the polarimetric information.
\end{abstract}

Index Terms - SAR, SAR Polarimetry, Speckle filtering, Binary Partition Tree, Segmentation

\section{INTRODUCTION}

SAR Polarimetry (PolSAR) has demonstrated, specially during the last decade, its significance for the analysis and the characterization of the Earth surface, as well as for the quantitative retrieval of biophysical and geophysical parameters. A set of complex radar scattered echoes are coherently processed in order to achieve high spatial resolution. As a consequence, the received signal is affected by a speckle term. Despite speckle is determined by the scattering process itself, its complexity makes necessary to consider it from a stochastic point of view and then, to assume the speckle term as a noise term. The speckle is a handicap in SAR imagery processing and information extraction and consequently some speckle filtering process is needed.

SAR and PolSAR data are non stationary as they reflect the complexity of the environment. Assuming that all the stochastic processes involved in the filtering process are ergodic, PolSAR filters must adapt to this non stationarity. Most recent state-of-the-art filtering techniques [1][2] are based on this approach, tending to define a different homogeneity neighborhoods for each image pixel. In this paper, we propose to tackle this issue by relying on a region-based multi-scale representation of the image by means of a Binary Partition Tree (BPT) and to perform filtering or segmentation tasks directly by pruning the BPT.

The authors thank DLR for providing the PolSAR data. This work has been supported by the Ramón-y-Cajal programm and the TEC200765690/TCM Spanish MICINN project.

\section{BINARY PARTITION TREE}

The Binary Partition Tree (BPT) was introduced in [3] as a region-based and multi-scale image representation. It contains information about image structure at different detail levels in a tree. Each node represents a region of the image. The tree leaves correspond to single pixels of the image and the remaining nodes represent the merging of the two child regions. Finally, the root node represents the whole image. The tree edges describe the inclusion relationship between nodes. From the leaves to the root, many regions with different sizes may be found. This multi-scale representation contains a lot of information about the image structure and may be exploited for PolSAR filtering and segmentation and for many other applications.

The BPT construction process can be performed in an efficient manner by an iterative algorithm [3] in a bottom-up approach. In the initial state, every pixel of the image will conform a single region. Iteratively the two most similar neighbor regions will be merged until a single region is achieved, keeping full track of the merging sequence within the tree structure. Thus, in order to be able to construct a BPT representation from a PolSAR image, the following elements have to be defined:

1. A region model: traditionally, under the complex Gaussian PolSAR model, the $3 \times 3$ estimated covariance matrix $\mathbf{Z}$ is employed to measure the region polarimetric information

$$
\mathbf{Z}=\left\langle\mathbf{k} \mathbf{k}^{H}\right\rangle_{n}=\frac{1}{n} \sum_{i=1}^{n} \mathbf{k}_{i} \mathbf{k}_{i}^{H}
$$

where $\mathbf{k}_{i}$ represents the scattering vector of the i-th pixel and $n$ represents the region size in pixels.

2. A similarity measure on the region model space to compare two neighboring regions $d(X, Y)$. The revised Wishart distance measure [4] $d_{w}$ is based on a statistical test assuming Wishart distributions and that one region statistics are known. However, since this measure is not symmetric, a modified symmetric version 
will be applied

$d_{s w}(X, Y)=\left(\operatorname{tr}\left(\mathbf{Z}_{X}^{-1} \mathbf{Z}_{Y}\right)+\operatorname{tr}\left(\mathbf{Z}_{Y}^{-1} \mathbf{Z}_{X}\right)\right) \cdot\left(n_{x}+n_{y}\right)$

where $\mathbf{Z}_{X}$ and $\mathbf{Z}_{Y}$ represent the estimated covariance matrices for regions $X$ and $Y$, respectively, and $n_{x}$ and $n_{y}$ represent their number of pixels.

For comparison purposes a new version of the symmetric revised Wishart dissimilarity will be used, only taking into account the diagonal elements of the $\mathbf{Z}$ matrix and assuming all off-diagonal values equal to zero

$$
d_{d w}(X, Y)=\left(\sum_{i=1}^{3}\left(\frac{Z_{X}^{2}+Z_{Y i i}^{2}}{Z_{X i i} Z_{Y i i}}\right)\right) \cdot\left(n_{x}+n_{y}\right)
$$

where $Z_{X i j}$ and $Z_{Y i j}$ represent the (i,j)th element of the estimated covariance matrices $\mathbf{Z}_{X}$ and $\mathbf{Z}_{Y}$, respectively.

\section{BPT PRUNING}

As stated in [3] an image segmentation may be obtained performing a tree pruning over the BPT. This pruning process is application dependent. In this paper two BPT pruning criteria are proposed focused on the speckle filtering application.

- Pruning based on the region number. The number of regions $n_{r}$ of the segmentation is fixed and the $n_{r}$ most different regions of the tree are obtained. If this decision relies on the same similarity measure employed for the BPT construction, then it is equivalent to stop the BPT construction process when $n_{r}$ regions are obtained.

- Homogeneity based pruning. A new homogeneity criteria is introduced to evaluate the region homogeneity. The main goal is to obtain a set of regions from the BPT that represent the largest homogeneous regions on the image.

The pruning based on the region number has the advantage of being simpler and faster than the homogeneity based pruning, since no new criteria nor evaluation are needed for pruning. However, in practical situations, it is almost impossible to fix a priori the optimum number of regions for a given PolSAR image.

For the homogeneity based pruning, the following homogeneity criteria is proposed

$$
\phi_{R}(X)=\frac{1}{n_{x}} \sum_{i=1}^{n_{x}} \frac{\left\|\mathbf{X}^{i}-\mathbf{Z}_{X}\right\|_{F}^{2}}{\left\|\mathbf{Z}_{X}\right\|_{F}^{2}}
$$

where $\mathbf{X}^{i}$ is the estimated covariance matrix for the $\mathrm{i}$-th pixel within region $X$ and $\|\cdot\|_{F}$ represents the Frobenius matrix norm.
Note that the homogeneity measure (4) depends on the values of all the region pixels and not only on the region model, as the similarity measure. The measure (4) may be interpreted as the information loss when modeling all the region pixels by its estimated covariance matrix. Thus, in order to determine if a region is homogeneous or not, a maximum value for the homogeneity measure will be defined, called pruning threshold.

\section{RESULTS}

To be able to make a quantitative evaluation of the proposed BPT pruning processes, a simulated $128 \times 128$ pixels PolSAR image is proposed in Fig. 1. Simulated data have been generated using the complex Gaussian PolSAR pdf assuming a reflection symmetric target

$$
\mathbf{C}=\sigma_{H H}\left(\begin{array}{ccc}
1 & 0 & \rho \sqrt{\gamma} \\
0 & \varepsilon & 0 \\
\rho^{*} \sqrt{\gamma} & 0 & \gamma
\end{array}\right)
$$

where ${ }^{*}$ denotes complex conjugate.

Two sets of images has been generated according to (5) with values $\gamma_{i}=1$ and $\varepsilon_{i}=0.1$ and variations for $\sigma_{H H i}$ and $\rho_{i}$ in different regions $i=1 \ldots 4$ as denoted in Fig. 1a

1. Variations in correlation: $\rho=\left\{0,0.25 e^{j \pi},-\right.$ $\left.0.5,0.75 e^{-j \pi}\right\} ; \sigma_{H H i}=1$

2. Variations in both correlation and intensity: $\rho=$ $\left\{0,0.25 e^{j \pi},-0.5,0.75 e^{-j \pi}\right\} ; \sigma_{H H}=\{1,9,25,49\}$

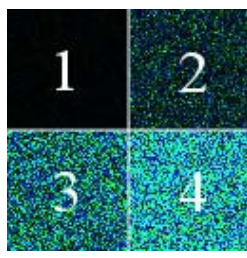

(a)

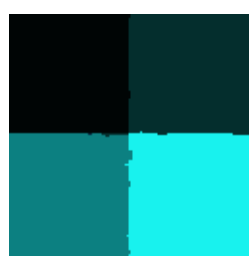

(b)

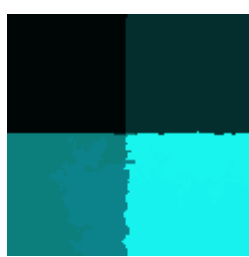

(c)
Fig. 1: Simulated image with variations in both correlation and intensity. $C_{22}, C_{33}$ and $C_{11}$ are assigned to RGB channels. (a) Zone numeration; BPT pruning at $-6 \mathrm{~dB}$ with (b) $d_{s w}$ and (c) $d_{d w}$.

To assess qualitatively the goodness of the processed image $X$ compared with the ground truth $Y$ a relative error measure is used

$$
E_{R}(X, Y)=\frac{1}{n_{h} \cdot n_{w}} \sum_{i=1}^{n_{h}} \sum_{j=1}^{n_{w}} \frac{\left\|\mathbf{X}^{i j}-\mathbf{Y}^{i j}\right\|_{F}^{2}}{\left\|\mathbf{Y}^{i j}\right\|_{F}^{2}}
$$

where $n_{h}$ and $n_{w}$ are the image height and width in pixels, respectively, $\mathbf{X}^{i j}$ represents the $(i, j)$ th pixel value of image $X$. 

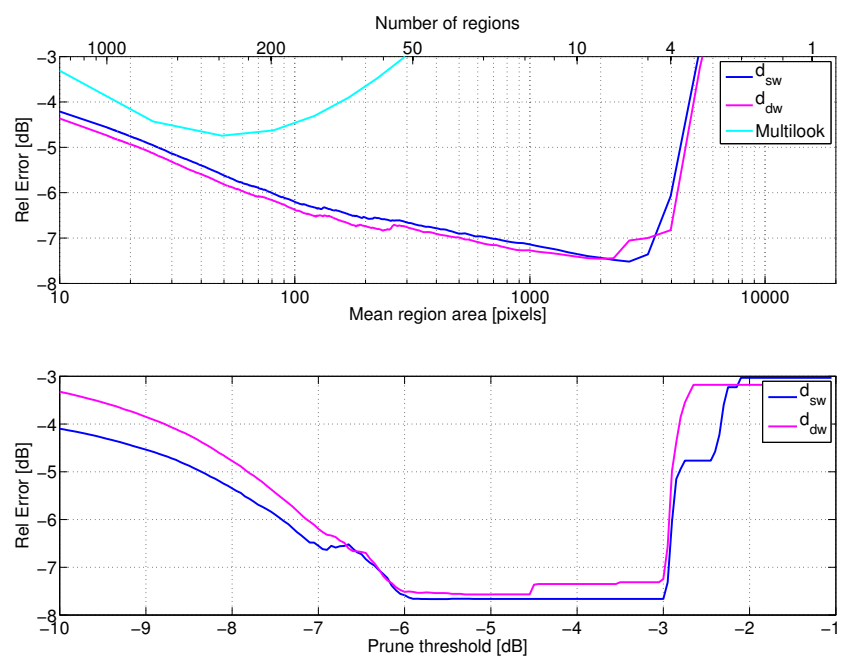

Fig. 2: Relative error for simulated images with variations in both correlation and intensity. BPT number of regions based pruning (top) and homogeneity based pruning (down).
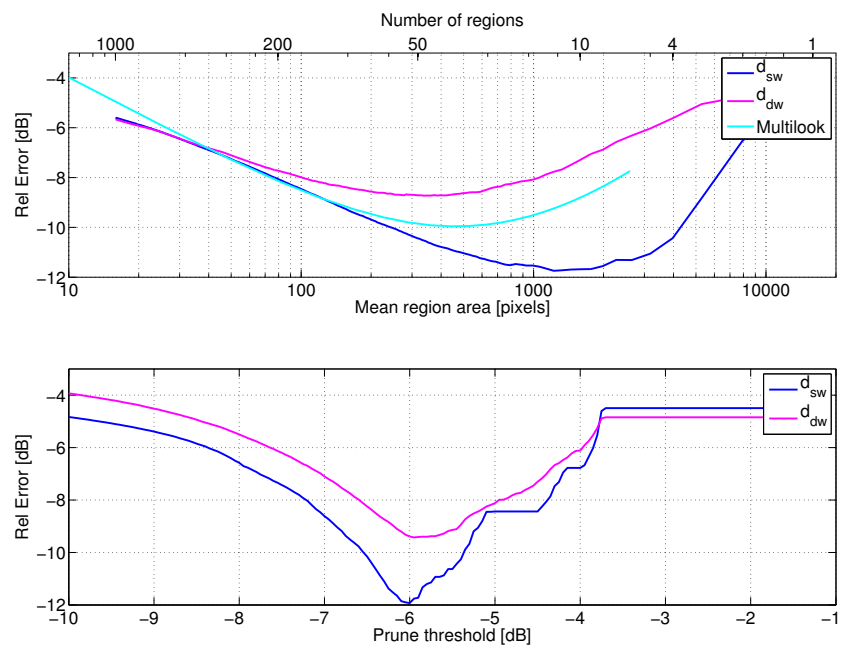

Fig. 3: Relative error for simulated images with variations in correlation. BPT number of regions based pruning (top) and homogeneity based pruning (down).

Note that the relative error measure (6) is based on the inverse signal to noise ratio $\left(S N R^{-1}\right)$ averaged for all the image pixels.

Fig. 2 shows the relative error over the image with variations in both correlation and intensity for the two proposed BPT pruning strategies. Fig. 3 shows the relative error over the image with variations in correlation only. The upper plot of the figures corresponds to the pruning based on the region number and the lower plot corresponds to region homogeneity based pruning. As observed in Fig. 2, in all the cases the BPT-filtering approach obtains lower values that the multilook, specially for large areas, without the alteration of the

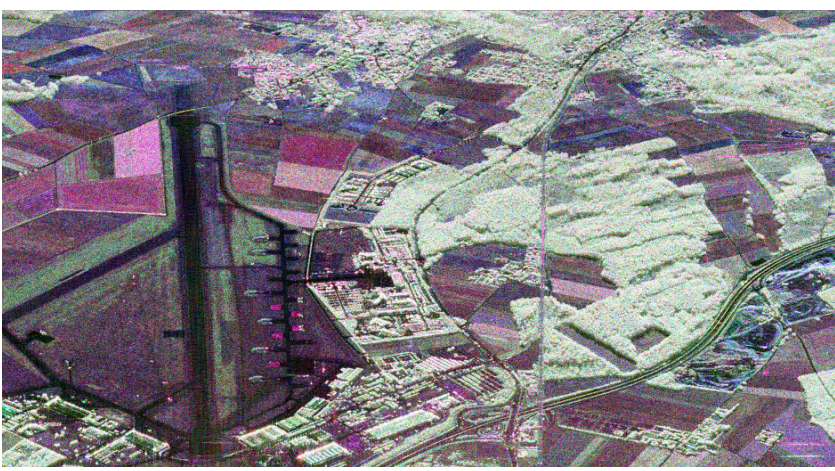

(a)

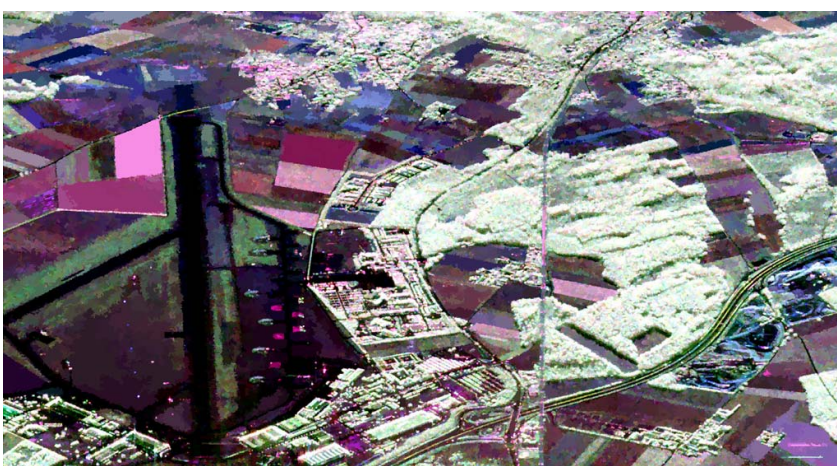

(b)

Fig. 4: Pauli RGB images: (a) original and (b) processed.

image contours. When there are variations only in the offdiagonal matrix elements, as in Fig. 3, the $d_{d w}$ similarity measure is not sensitive to contours and only $d_{s w}$ can exploit all the polarimetric information and improve the multilook filtering.

Complementarily, the application of the BPT-filtering strategy for PolSAR filtering has been considered with experimental PolSAR data that was acquired in a measurement campaign conducted by the DLR in 1999 with its experimental SAR system, E-SAR, over the Oberpfaffenhofen test-site, southern Germany. Data were collected at L-band, with a spatial resolution of $1.5 \mathrm{~m} \times 1.5 \mathrm{~m}$ in fully polarimetric mode.

Fig. 4 presents the original Pauli RGB image of the previous data set, compared with Pauli RGB image obtained from the speckle filtered data set considering the previous BPT approach. In this case, the distance $d_{s w}$ has been considered for the construction process and the homogeneity based pruning with a pruning threshold of $-2 \mathrm{~dB}$ has been assumed. As one may observe, the BPT-based filtering does not introduce neither new contours nor artifacts on the filtered data set. It is worth to mention that the proposed BPT-filtering approach makes it possible to retain almost the original spatial resolution in case of point and man-made targets, whereas it allows a large filtering in case of distributed targets such agricultural and forest areas. 


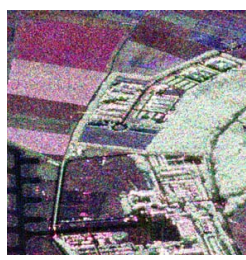

(a)

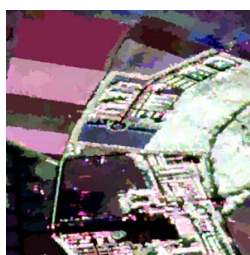

(c)

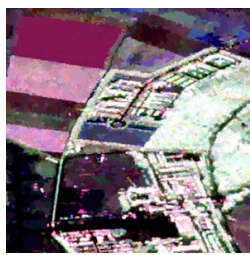

(b)

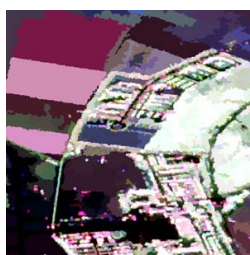

(d)
Fig. 5: Detail Pauli RGB images: (a) original and processed with pruning threshold (b) $-2 \mathrm{~dB}$ (c) $-1 \mathrm{~dB}$ and (d) $0 \mathrm{~dB}$.

As it has been indicated, one of the main features of the BPT-based filtering approach is the capability to perform a multi-scale analysis. Fig. 5 demonstrates this capability by changing the pruning threshold from $-2 \mathrm{~dB}$ to $0 \mathrm{~dB}$. As observed, the multi-scale capability allows different levels of filtering, specially on homogeneous areas, but without the introduction of new contours or artifacts. This feature allows to introduce strong filtering in case of homogeneous areas whereas maintaining the spatial resolution and details of the data.

Additionally to the maintenance of the spatial resolution and spatial details of the image, Figs. 4 and 5 also shown that the BPT-filtering approach is able to maintain the polarimetric information, as the Pauli representations do not vary between the original and the filtered images. As indicated, the model for the nodes of the BPT consists of (1), which represents the Maximum Likelihood Estimation of the covariance matrix to represent the data with the complex Gaussian pdf. Consequently, no global biases are introduced in the filtered signal since given a segmentation, a pixel can only belong to one region. In order to demonstrate the capability to retain the polarimetric information, the parameters of the eigendecomposition of the covariance matrix, namely, Entropy $(\mathrm{H})$, Anisotropy (A) and the average alpha angle $\bar{\alpha}$ have been obtained with a $7 \times 7$ multilook filters and compared with the ones obtained through the BPT-filtering approach. As observed in Fig. 6, both approaches obtain qualitatively the same values. Nevertheless, the BPT-approach is able to obtain these parameters with larger filtering in case of distributed targets reducing estimation errors. additionally, in case of distributed scatterers, the BPT-filtering approach is able to maintain the spatial resolution. Consequently, the proposed filtering approach improves the estimation of the polarimetric information, both, in point as well as in distributed scatterers.

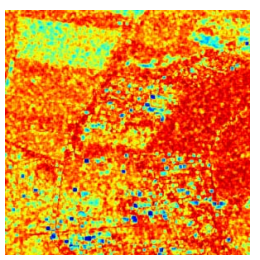

(a) $\mathrm{H}$

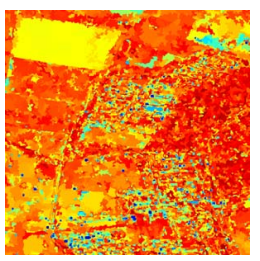

(d) $\mathrm{H}$

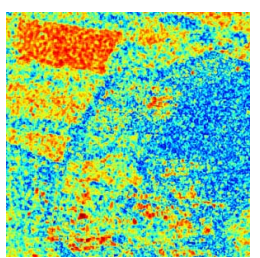

(b) $\mathrm{A}$

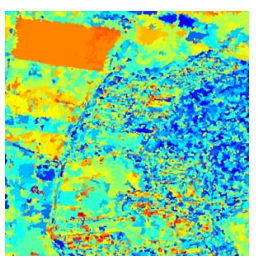

(e) $\mathrm{A}$

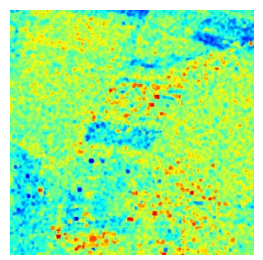

(c) $\bar{\alpha}$

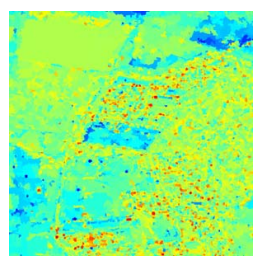

(f) $\bar{\alpha}$
Fig. 6: $\mathrm{H} / \mathrm{A} / \bar{\alpha}$ with $7 \times 7$ multilook (a), (b) and (c), and with BPT employing a pruning threshold of $-2 \mathrm{~dB}(\mathrm{~d})$, (e) and (f).

\section{CONCLUSIONS}

This paper proposes a novel multi-scale polarimetric speckle filtering strategy, based on a BPT representation of the data, that is able to maintain spatial resolution and details in case of point scatterers, while introducing large filtering in case of homogeneous ones. This filtering strategy adapts to the complete covariance matrix which represents the main difference with respect to previous approaches that only rely on its diagonal elements. As demonstrated, the proposed strategy also maintains the polarimetric information without the introduction of biases.

Furthermore, it has been proved that the BPT representation can be employed to extract useful information in PolSAR images, and it can be employed for many other applications.

\section{REFERENCES}

[1] Jong-Sen Lee, M. R. Grunes, and G. de Grandi, "Polarimetric sar speckle filtering and its implication for classification," IEEE TGRS, vol. 37, no. 5, pp. 2363-2373, 1999.

[2] G. Vasile, E. Trouve, Jong-Sen Lee, and V. Buzuloiu, "Intensitydriven adaptive-neighborhood technique for polarimetric and interferometric sar parameters estimation," IEEE TGRS, vol. 44, no. 6, pp. 1609-1621, 2006.

[3] P. Salembier and L. Garrido, "Binary partition tree as an efficient representation for image processing, segmentation, and information retrieval," IEEE TIP, vol. 9, no. 4, pp. 561-576, 2000.

[4] P. R. Kersten, Jong-Sen Lee, and T. L. Ainsworth, "Unsupervised classification of polarimetric synthetic aperture radar images using fuzzy clustering and em clustering," IEEE TGRS, vol. 43, no. 3, pp. 519-527, 2005. 